\title{
Examining the Mechanical Equilibrium of Microscopic Stresses in Molecular Simulations
}

\author{
Alejandro Torres-Sánchez, Juan M. Vanegas, ${ }^{*}$ and Marino Arroyo ${ }^{\dagger}$ \\ LaCàN, Universitat Politècnica de Catalunya BarcelonaTech, 08034 Barcelona, Spain
}

\begin{abstract}
The microscopic stress field provides a unique connection between atomistic simulations and mechanics at the nanoscale. However, its definition remains ambiguous. Rather than a mere theoretical preoccupation, we show that this fact acutely manifests itself in local stress calculations of defective graphene, lipid bilayers, and fibrous proteins. We find that popular definitions of the microscopic stress violate the continuum statements of mechanical equilibrium, and we propose an unambiguous and physically sound definition.
\end{abstract}

The stress $\boldsymbol{\sigma}(\boldsymbol{x})$ is a second-rank tensor field encoding the internal force distribution in a continuum system; when multiplied by a unit vector $\boldsymbol{n}$, it provides the forces per unit area in the material across a surface passing through $\boldsymbol{x}$ and perpendicular to $\boldsymbol{n}$. The continuum stress field can be connected to the statistical mechanics of discrete particle systems, in what is called the microscopic stress. Today, the microscopic stress is increasingly used to recapitulate mechanical information contained in long molecular dynamics (MD) trajectories of nonuniform systems, or to connect molecular details with continuum physics at larger scales. Significant applicationsinclude defective bulk [1,2] and two-dimensional crystals [3,4], biomolecular assemblies such as lipid bilayers [5-7], membrane proteins [8,9], and even isolated molecules [10].

The average (or virial) stress of a periodic system can be uniquely defined and given a precise thermodynamical interpretation [11]. There are, however, multiple procedures to map a statistical mechanics ensemble into a stress field. This indeterminacy is expected since any divergence-free symmetric tensor field (self-equilibrated) can be added to the local stress without affecting the continuum statements of balance of linear and angular momentum for a system in equilibrium [12,13],

$$
\begin{gathered}
\sigma_{i j, j}=0, \\
\sigma_{i j}=\sigma_{j i},
\end{gathered}
$$

where we have ignored externally applied force and torque densities. The ambiguity in the microscopic stress is widely appreciated theoretically, but its practical consequences have been largely overlooked, partly because different definitions coincide for simple force fields [7]. As molecular simulations model increasingly complex systems, the issue of whether different definitions of the microscopic stress satisfy the fundamental requirements of mechanical equilibrium in Eq. (1) has not been systematically examined.

Here, we simulate the equilibrium behavior of three important condensed matter systems-defective graphene, lipid bilayers, and coiled-coil fibrous proteins-using MD simulations with realistic atomistic force fields, and study the significance of the definition of the microscopic stress. We consider the atomic virial stress $[3,4,14]$ and several flavors of the Irving-Kirkwood stress [7,8,15-19]. Strikingly, we find that the atomic virial stress does not satisfy balance of linear momentum. Furthermore, we find that a widely used version of the Irving-Kirkwood stress [15] does not satisfy balance of angular momentum for systems with chiral constituents. Our results favor a recent canonical definition of the Irving-Kirkwood stress [16,17], which, however, is limited to multibody potentials with at most four-body interactions. Motivated by higher-body force fields used in materials science and biochemistry [20,21], we develop and test a new and general procedure [22] to uniquely obtain a physically meaningful microscopic stress.

Microscopic stress fields include a kinetic contribution $\sigma_{K}$, describing the flux of momentum due to internal vibrations, and a potential contribution $\sigma_{V}$, accounting for the interatomic forces. Since the difficulty in the microscopic stress definition stems from the potential part, we focus only on this contribution and refer to [23] for the expression of $\boldsymbol{\sigma}_{K}$. For solid systems, where atoms stay relatively immobile, it is common to resort to the atomic virial stress definition [14], which is conceptually and computationally simpler than other definitions. In this method, the potential component is computed by distributing the virial of each potential contribution equally among the particles involved. More specifically, let the potential of the system be described additively as $V=\sum_{I=1}^{M} V_{I}$, with each contribution involving $n_{I}$ particles. Then, we have

$$
\sigma_{V}^{\alpha}=\frac{1}{\Omega_{\alpha}} \sum_{I \in \mathcal{I}^{\alpha}} \frac{1}{n_{I}}\left\langle\sum_{\beta} \frac{\partial V_{I}}{\partial \boldsymbol{r}^{\beta}} \otimes \boldsymbol{r}^{\beta}\right\rangle,
$$

where $\boldsymbol{r}^{\beta}$ is the position of particle $\beta, \mathcal{I}^{\alpha}$ collects the set of potential contributions involving particle $\alpha$, and $\Omega_{\alpha}$ is the volume of this particle.

An alternative definition of the microscopic stress with a more solid statistical mechanics foundation was pioneered by Irving and Kirkwood [34,35] for two-body potentials. This approach, which we label as IK, defines the stress at 
each point in space, $\boldsymbol{\sigma}(\boldsymbol{x})$, rather than at the particles, and is thus more popular in fluid systems. The IK microscopic stress is identified by invoking Eq. (1a), which is therefore satisfied $a b$ initio $[34,35]$. The potential component at position $\boldsymbol{x}$ is given by

$$
\sigma_{V}(x)=\left\langle\sum_{\alpha, \beta>\alpha} \boldsymbol{f}^{\alpha \beta} \otimes \boldsymbol{r}^{\alpha \beta} B\left(\boldsymbol{r}^{\alpha}, \boldsymbol{r}^{\beta} ; \boldsymbol{x}\right)\right\rangle,
$$

where $\boldsymbol{r}^{\alpha \beta}=\boldsymbol{r}^{\beta}-\boldsymbol{r}^{\alpha}, B\left(\boldsymbol{r}^{\alpha}, \boldsymbol{r}^{\beta} ; \boldsymbol{x}\right)=\int_{0}^{1} \delta\left[(1-s) \boldsymbol{r}^{\alpha}+s \boldsymbol{r}^{\beta}-\boldsymbol{x}\right] d s$ is the bond function [35], and $\delta\left(\boldsymbol{r}^{\alpha}-\boldsymbol{x}\right)$ is the Dirac delta distribution centered at $\boldsymbol{x}$. For two-body interactions $V_{I}=V_{\alpha \beta}\left(r^{\alpha \beta}\right)$, we have $\boldsymbol{f}^{\alpha \beta}=V_{\alpha \beta}^{\prime} \boldsymbol{r}^{\alpha \beta} / r^{\alpha \beta}$, where $r^{\alpha \beta}=$ $\left|\boldsymbol{r}^{\alpha \beta}\right|$. In practice, space is subdivided into a grid and the microscopic stress is evaluated at discrete positions [7].

There have been a number of extensions of the IK framework to deal with multibody potentials $[12,15,16,18,19]$. Some of these approaches fully retain balance of linear momentum by construction $[12,15,16]$, by defining $f^{\alpha \beta}$ as the terms of a pairwise force decomposition $\boldsymbol{F}^{\alpha}=\sum_{\beta}{ }^{\alpha \beta}$ satisfying $\boldsymbol{f}^{\alpha \beta}=-f^{\beta \alpha}$, with $\boldsymbol{F}^{\alpha}$ being the total force acting on particle $\alpha$. The indeterminacy of the local stress tensor manifests itself in the IK definition because the pairwise force decomposition is not unique for potentials involving three or more particles. We consider initially the

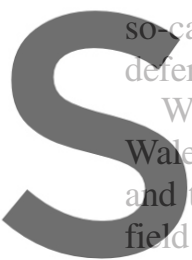
alled central force decompos further discussion to later i We first consider a periodic s defect as a model system the IK stresses. The system
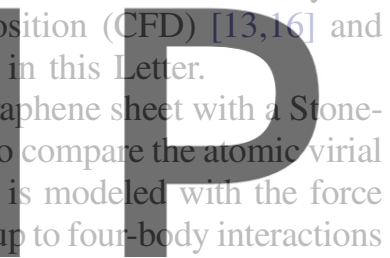

and simulated in a NVT ensemble at $300 \mathrm{~K}$ [23]. We compute microscopic stresses here and elsewhere in the Letter with a

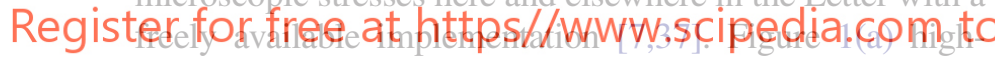

lights the fundamental features of each stress definition. The atomic virial stress is defined discretely at the atoms, while the IK stress is continuous but exhibits marked concentrations along lines joining atomic positions, a signature of the force decomposition. It is often convenient to spatially average the discrete features in these fields with weighting functions such as Gaussian kernels [13,23,38]. Figure 1(b) shows that the spatially averaged fields $\sigma$ according to both notions of stress are qualitatively similar, although the atomic virial stress exhibits smaller magnitudes.

We analyze next whether these fields are in equilibrium, as physically expected. Importantly, it can be shown by a simple calculation that $\sigma_{i j, j}(\boldsymbol{x})$ is the spatial average of $\sigma_{i j, j}(\boldsymbol{y})$ [23]. Therefore, $\sigma_{i j, j}$ should be zero if the corresponding microscopic stress $\sigma_{i j}$ obeys Eq. (1a). Strikingly, the atomic virial stress is strongly out of equilibrium in the vicinity of the defect, Fig. 1(c). In contrast, the IK stress field exhibits nearly zero divergence (except from deviations due to discretization and limited statistical sampling). Thus, our results suggest that the atomic virial stress should be employed only for visualization purposes.

We focus next on the various extensions of the IK stress for multibody interactions. A generalization of the method

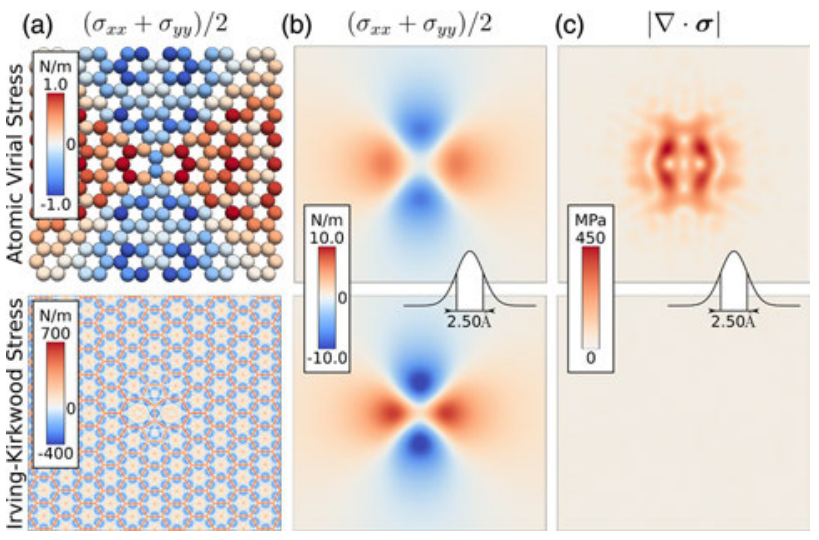

FIG. 1 (color online). Balance of linear momentum of the microscopic stress in a graphene sheet with a Stone Wales defect: comparison of the atomic virial (upper row) and the IK (lower row) stress definitions. (a) Trace of the raw stress fields. (b) Spatially averaged trace of the stress field. (c) Norm of the divergence of the spatially averaged microscopic stress. Plots focus on a small region near the defect in the $x-y$ plane. Because the system is quasi 2D, we only consider the in plane compo nents and express stress in units of surface tension.

of planes has been proposed by Heinz, Paul, and Binder (HPB) $[18,19]$, which is consistent with the IK stress for

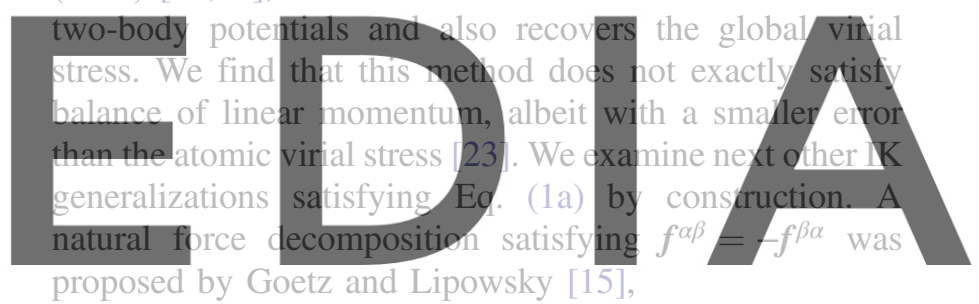

\section{download the versioin without the watermark}

This Goetz-Lipowsky decomposition (GLD) has been widely employed to analyze MD simulations ([5,7,8] and references therein), yet it produces noncentral forces; i.e., in general $\boldsymbol{f}_{\mathrm{GLD}}^{\alpha \beta}$ is not parallel to $\boldsymbol{r}^{\alpha \beta}$. In principle, this could lead to nonsymmetric stresses $[12,16]$. Recently, a central force decomposition has been proposed by Admal and Tadmor $[13,16]$, which produces a symmetric stress tensor by construction because $\boldsymbol{f}^{\alpha \beta}$ is defined such that it is always parallel to $\boldsymbol{r}^{\alpha \beta}$, see Eq. (3). By invariance with respect to rigid body transformations, the additive potential contributions can be represented in terms of interatomic distances, $\left.\tilde{V}_{I}\left(r^{12}, \ldots, r^{\left(n_{I}\right.} 1\right), n_{I}\right)=V_{I}\left(\boldsymbol{r}^{1}, \ldots, \boldsymbol{r}^{n_{I}}\right)$, leading to the CFD pairwise forces

$$
f_{\mathrm{CFD}}^{\alpha \beta}=\sum_{I=1}^{M} \frac{\partial \tilde{V}_{I}}{\partial r^{\alpha \beta}} \frac{\boldsymbol{r}^{\alpha \beta}}{r^{\alpha \beta}} .
$$

The CFD and GLD force decompositions result in pairwise forces with large differences in magnitude and direction [23]. To explore the features of each force decomposition, we consider a lipid bilayer system of fluid DPPC 
(1,2-dipalmitoyl-sn-glycero-3-phosphocholine), which is isotropic in the membrane plane $(x-y)$ at the simulated temperature $(323 \mathrm{~K})$. In addition to the conventional profiles of all stress components across the thickness, we adopt an unconventional but intuitive method to visualize stress through the traction vector, $\boldsymbol{t}=\boldsymbol{\sigma} \cdot \boldsymbol{n}$, on a given internal surface with unit normal $\boldsymbol{n}$ (Fig. 2). The traction can be decomposed into a normal and a tangential component, $\boldsymbol{t}=t_{n} \boldsymbol{n}+\boldsymbol{\tau}$. Here, we consider a test cylinder, and represent the normal traction $t_{n}$ as a color map and the tangential component $\boldsymbol{\tau}$ using arrows.

In agreement with the symmetries of this system and the fluidity of the bilayer, the CFD stress is diagonal with equal lateral components $\left(\sigma_{x x}=\sigma_{y y}\right)$ and a normal constant component across the bilayer $\left[\sigma_{z z}(z)=\right.$ const.] as dictated by Eq. (1a) [Fig. 2(a)]. We note that for a bilayer in the gel phase, the off-diagonal components could be nonzero but should nevertheless respect the symmetry of $\sigma$. In sharp contrast, the GLD stress exhibits nonzero in-plane off-diagonal components, which are antisymmetric $\left(\sigma_{x y}=-\sigma_{y x}\right)$ and of significant magnitude [Fig. 2(b)], hence violating Eq. (1b). Furthermore, CFD and GLD produce significantly different lateral components, and, hence, normal tractions $\left(t_{n}=\sigma_{x x}=\sigma_{y v}\right)$ [Fig. 2(c)].

Focusing on the tangential traction, we note that $\tau$ is

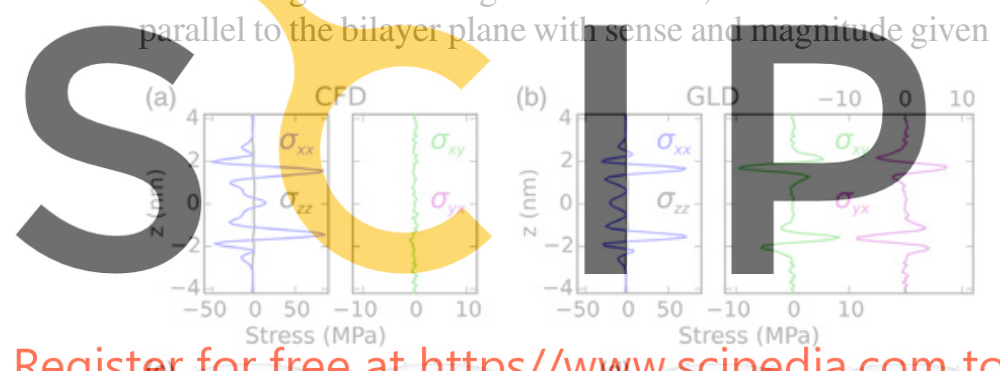

Register for free at https//www.scipedia.com to

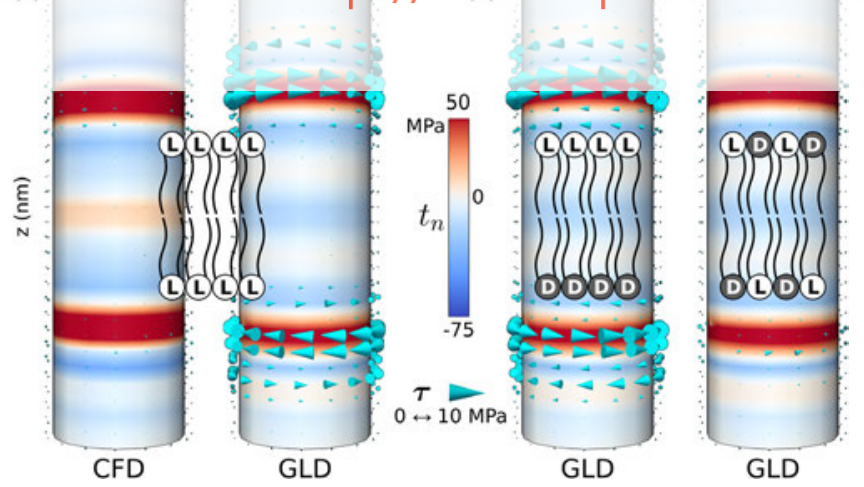

FIG. 2 (color online). Balance of angular momentum of the IK stress in a planar DPPC fluid membrane, and influence of lipid chirality. In plane components of the stress tensor analyzed with CFD (a) and GLD (b). (c) Visualization of the normal and tangential components of the traction vector along a cylindrical surface perpendicular to the bilayer plane, for both CFD and GLD. The effect of the lipid chirality on the GLD stress for the two DPPC enantiomers, L DPPC and D DPPC, is shown in (d), where we consider a lipid membrane composed of monolayers with different chiralities (one with pure L DPPC and the other with pure D DPPC, left), and a bilayer with both monolayers having equal numbers of L DPPC and D DPPC (right). by $\sigma_{x y}=-\sigma_{y x}$. As expected, for CFD $\tau$ is nearly zero. For GLD, however, it is clear from Fig. 2(c) that the nonsymmetry of the stress tensor introduces distributed torques of opposite sign in each leaflet of the bilayer, since $\sigma_{x y}(z)=-\sigma_{x y}(-z)$. We hypothesize that such behavior may be due to the internal structure of each lipid, since the headgroup portion of DPPC contains a chiral carbon. We test this hypothesis by comparing the stress tensors for three systems with different mixtures of the two DPPC enantiomers (L-DPPC and D-DPPC). Consistent with this hypothesis, the torques induced in each monolayer according to GLD adopt the same sign for a system with one monolayer composed solely of L-DPPC and the second monolayer composed solely of D-DPPC [Fig. 2(d)]. Mixing equal numbers of each chiral lipid in both monolayers results in nearly zero distributed torques according to the GLD stress. Thus, the off-diagonal components of the GLD stress tensor reflect the average chirality of the molecular composition. In contrast, we find that the CFD stress tensor is essentially unchanged by the lipid chirality [23].

To physically interpret the GLD distributed torques, it is necessary to resort to an extended theory of continuum mechanics. In micropolar continuum theories, these torques can be balanced locally by invoking a couple stress field $m$,

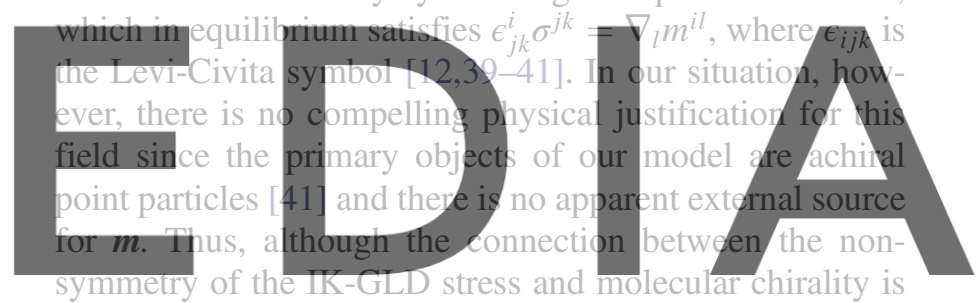

very appealing, this example undermines its mechanical doviploaidnthe vers ionswithlouth the watermark metric stresses very similar to GLD for this system [23].

The microscopic stress tensor not only serves as a tool to explore the local distribution of forces, but can also provide important material properties. For instance, the Gaussian curvature elastic modulus of lipid bilayers can be computed as $\kappa=\int\left[\left(\sigma_{x x}+\sigma_{y y}\right) / 2-\sigma_{z z}\right] z^{2} d z$, which is highly sensitive to the features of the stress profile (see [6] and references therein). For the three bilayer systems with different chiralities in Fig. 2, we obtain $\kappa_{\mathrm{CFD}}=$ $(-6.4,-6.7,-6.1) \times 10^{20} \mathrm{~J}$, in agreement with the common estimates of $\kappa$ in the order of the negative of the bending modulus $\sim 5-15 \times 10^{20} \mathrm{~J}$ [42]. Strikingly, we find $\kappa_{\mathrm{GLD}}=(0.91,0.57,1.3) \times 10^{20} \mathrm{~J}$, with the wrong sign-suggesting that a DPPC bilayer would be unstable [43] — and widely varying magnitudes.

Taken together, these results show that the choice of microscopic stress definition is not a mere theoretical preoccupation. Our results strongly favor the IK-CFD definition, which, unlike the atomic virial or the IK-GLD stresses, identically satisfies Eq. (1) for a system in equilibrium. However, CFD is not uniquely defined when $n_{I}>4$. The geometric reason behind this ambiguity is that the $n_{I}\left(n_{I}+1\right) / 2$ interatomic distances $\left(r^{12}, \ldots, r^{\left(n_{I} 1\right), n_{I}}\right)$ 
involved in a given multibody potential $V_{I}$ cannot be arbitrarily chosen in $\mathcal{D}_{I}=\mathbb{R}_{+}^{n_{I}\left(n_{I}+1\right) / 2}$. There are geometric conditions that make these distances realizable by a system of $n_{I}$ particles, which define the so-called shape space $\mathcal{S}_{I} \subset \mathcal{D}_{I}$. When $n_{I}>4$, the dimension of the manifold $\mathcal{S}_{I}$ is smaller than $n_{I}\left(n_{I}+1\right) / 2$, and, therefore, the differential calculus involved in Eq. (5) needs to be carefully considered $[44,45]$. More practically, when $n_{I}>4$ there are infinitely many different ways to express the potential, $\tilde{V}_{I}$, in terms of interatomic distances, each resulting in a different force decomposition and microscopic stress $[13,16]$.

In the spirit of [46-48], we propose an alternative thermodynamic derivation of the IK microscopic stress, which naturally and unambiguously extends CFD to multibody potentials. In analogy to the Doyle-Ericksen equation of continuum mechanics $[49,50]$, the stress tensor can be defined from covariance arguments as

$$
\boldsymbol{\sigma}(\boldsymbol{x})=\frac{2}{\sqrt{ } g(\boldsymbol{x})} \frac{\delta A}{\delta \boldsymbol{g}(\boldsymbol{x})}
$$

where $g(\boldsymbol{x})$ is the Jacobian determinant of the metric and $\delta A$ is the variation of the canonical free energy with respect to an infinitesimal change of metric $\delta g(x)$ resulting from a change of coordinates. As fully detailed in [22], this

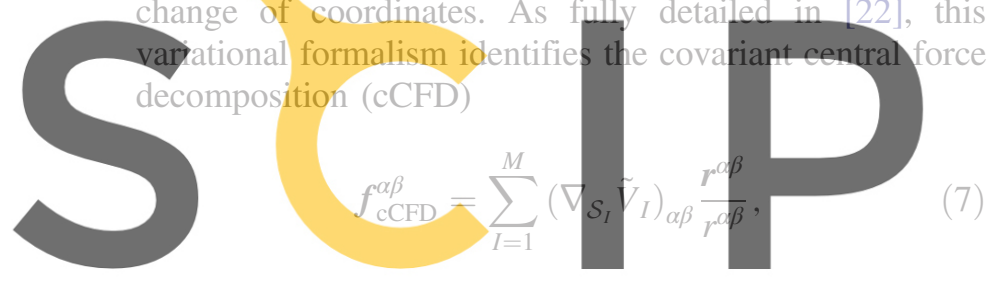

where $\nabla_{\mathcal{S}_{I}} \tilde{V}_{I}$ is the covariant derivative of the potential

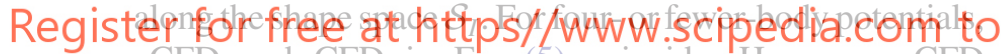
cCFD and CFD in Eq. (5) coincide. However, cCFD circumvents the main limitation of CFD by providing a unique expression for potentials with any number of particles. In practice, $\left(\nabla_{\mathcal{S}} \tilde{V}_{I}\right)_{\alpha \beta}$ can be computed by projecting $\partial \tilde{V}_{I} / \partial r^{\alpha \beta}$ for an arbitrary extension onto $\mathcal{S}_{I}$ [23]. Through a different rationale, the projection of the CFD onto the shape space has been recently discussed in [51]. Our assumption that the potential is additively decomposed into a many-body expansion is appropriate for most classical force fields. For semiempirical methods based on density functional theory concepts, such as the embedded-atom model [20], this additive structure is not apparent. We refer the interested reader to [22,51] for further discussion.

We test the cCFD microscopic stress by considering a coiled-coil structural protein, composed of two identical $\alpha$ helical chains that wrap around each other to form a superhelix. The coiled-coil structure is a double "zipper," with an inner core of intercalating hydrophobic amino acids that are flanked by opposing negatively and positively charged amino acids, Fig. 3(a). We model this system as an infinitely long periodic molecule with a widely used protein force field (CHARMM22/CMAP) [21,52] involving up to five-body interactions. We compare the tractions on the surface of the
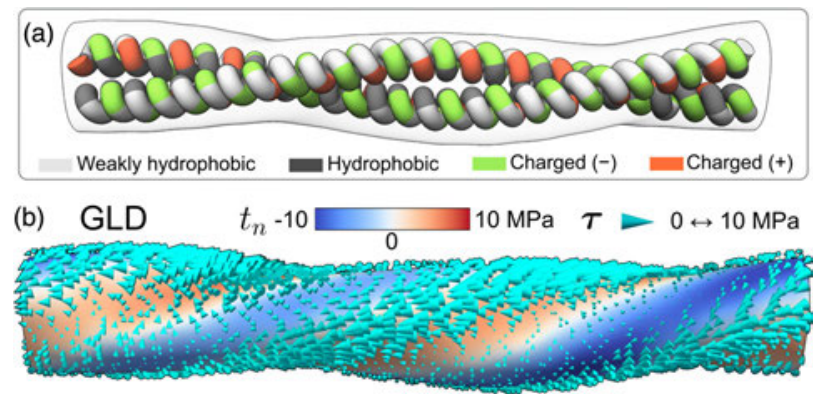

(c) $\mathrm{CCFD}$

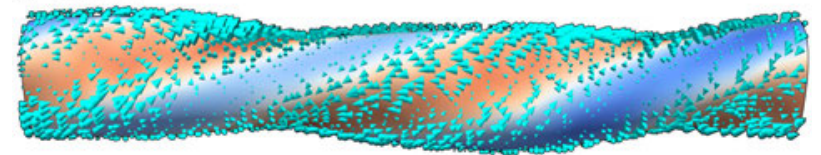

(d) $\mathrm{nCFD}$

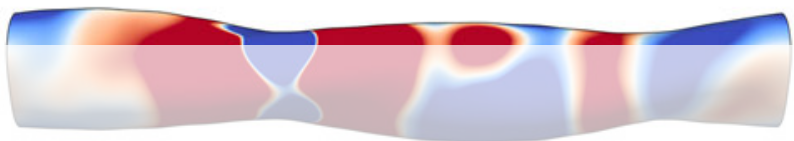

FIG. 3 (color online). The IK stress for force fields beyond four body interactions. (a) Ribbon representation of a structural coiled coil protein simulated with the five body CMAP potential (cross term energy correction map for adjacent dihedrals used with the CHARMM22 force field). Tractions at the surface of the protein are calculated with different variants of the IK stress

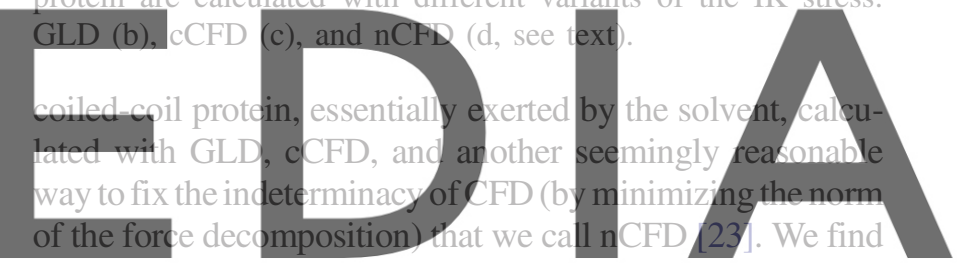

that for GLD and cCFD, $t_{n}$ exhibits a similar pattern that follows the left-handed helical structure, Figs. 3(b) and 3(c).

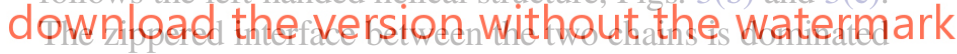

by outward tractions (red), which transition to inward tractions (blue) at the periphery of the protein. In contrast, nCFD produces spurious maps of $t_{n}$ [Fig. 3(d)], highlighting the need for a physically meaningful method to fix the gauge freedom of CFD [40]. Focusing on $\boldsymbol{\tau}$, cCFD presents tractions that locally equilibrate and do not produce net forces. GLD, however, produces a predominantly leftward traction field following the twist of the protein as a result of the nonsymmetric components of $\boldsymbol{\sigma}$. Quantitatively, the GLD tractions produce a net force per unit protein length along its axis of $34 \mathrm{mN} / \mathrm{m}$, whereas for cCFD we have $0.35 \mathrm{mN} / \mathrm{m}$. Thus, the GLD stress again contains information about chirality but produces tractions that cannot be physically balanced in our periodic system, which does not undergo any translation or rotation during the course of the simulation.

In summary, the ambiguity of the microscopic stress acutely emerges when analyzing complex materials and biomolecular assemblies. Strikingly, the widely used atomic virial stress does not satisfy balance of linear momentum, while a popular version of the Irving-Kirkwood stress does not satisfy balance of angular momentum as a result of molecular chirality. In contrast, Irving-Kirkwood stress fields based on a covariant central force decomposition can be 
rationally derived irrespective of the many-body nature of the potential and satisfy by construction mechanical equilibrium in Eq. (1). This definition of microscopic stress thus provides a solid footing to systematically connect MD simulations and the mechanical behavior of materials at the nanoscale $[6,53]$.

We acknowledge the support of the European Research Council under the European Community's seventh Framework Programme (FP7/2007-2013)/ERC Grant No. 240487, and the computer resources, technical expertise, and assistance provided by the Red Española de Supercomputación. A. T.-S. acknowledges the support of the Spanish government through Grant No. BES-2012054895. We thank Dr. Bogdan Costescu for assistance with the graphene force field parameters.

A. T.-S. and J. M. V. contributed equally to this work.

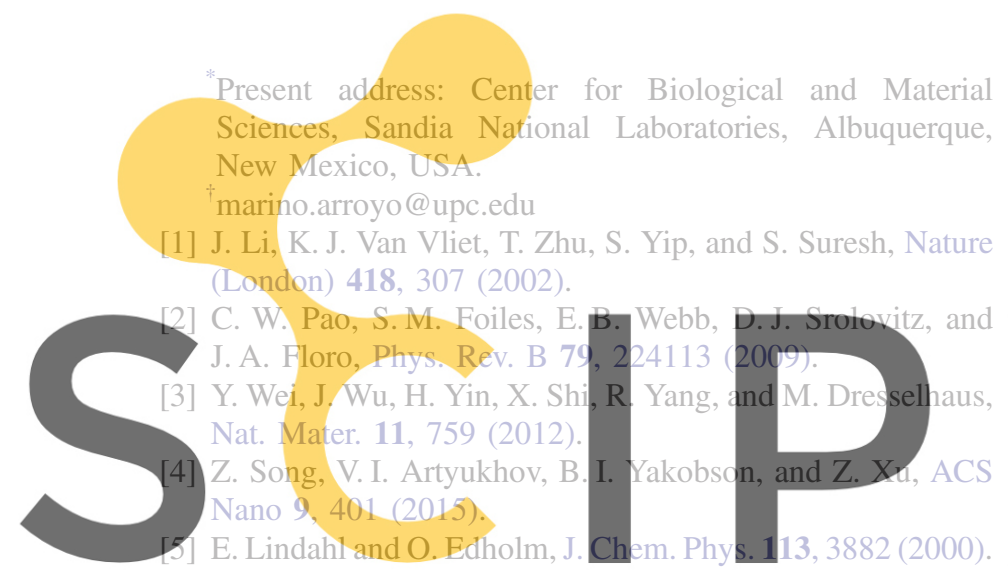

[6] M. Hu, D. H. de Jong, S. J. Marrink, and M. Deserno, Faraday Discuss. 161. 365 (2013

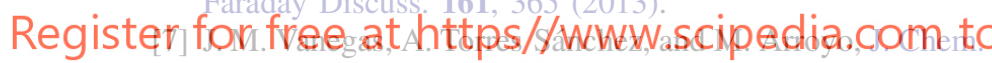
Theory Comput. 10, 691 (2014).

[8] O. H. S. Ollila, H. J. Risselada, M. Louhivuori, E. Lindahl, I. Vattulainen, and S. J. Marrink, Phys. Rev. Lett. 102, 078101 (2009).

[9] J. M. Vanegas and M. Arroyo, PLoS One 9, e113947 (2014).

[10] H. W. Hatch and P. G. Debenedetti, J. Chem. Phys. 137, 035103 (2012).

[11] D. H. Tsai, J. Chem. Phys. 70, 1375 (1979).

[12] P. Schofield and J. Henderson, Proc. R. Soc. A 379, 231 (1982).

[13] E. B. Tadmor and R.E. Miller, Modeling Materials: Continuum, Atomistic and Multiscale Techniques (Cambridge University Press, Cambridge, England, 2011).

[14] A. P. Thompson, S. J. Plimpton, and W. Mattson, J. Chem. Phys. 131, 154107 (2009).

[15] R. Goetz and R. Lipowsky, J. Chem. Phys. 108, 7397 (1998).

[16] N. C. Admal and E. B. Tadmor, J. Elast. 100, 63 (2010).

[17] N. C. Admal and E. B. Tadmor, J. Chem. Phys. 134, 184106 (2011).

[18] H. Heinz, W. Paul, and K. Binder, Phys. Rev. E 72, 066704 (2005).

[19] H. Heinz, Mol. Simul. 33, 747 (2007).

[20] M. S. Daw and M. I. Baskes, Phys. Rev. B 29, 6443 (1984).

[21] A. D. Mackerell, M. Feig, and C. L. Brooks, J. Comput. Chem. 25, 1400 (2004).
[22] A. Torres Sánchez, J. M. Vanegas, and M. Arroyo (to be published).

[23] See Supplemental Material at http://link.aps.org/ supplemental/10.1103/PhysRevLett.114.258102 for ex tended theory, figures, and information about simulation settings, which includes Refs. [24 33].

[24] B. Hess, C. Kutzner, D. van Der Spoel, and E. Lindahl, J. Chem. Theory Comput. 4, 435 (2008).

[25] S. Pronk, S. Páll, R. Schulz, P. Larsson, P. Bjelkmar, R. Apostolov, M. R. Shirts, J. C. Smith, P. M. Kasson, D. van der Spoel, B. Hess, and E. Lindahl, Bioinformatics 29, 845 (2013).

[26] O. Berger, O. Edholm, and F. Jähnig, Biophys. J. 72, 2002 (1997).

[27] D. P. Tieleman, Structures and topologies for biomembranes and membrane protein systems, http://wcm.ucalgary.ca/ tieleman/downloads.

[28] H. J. C. Berendsen, J. P. M. Postma, and W. F. van Gunsteren, in Intermolecular Forces, edited by B. Pullman (Reidel, Dordrecht, 1981), pp. 331342.

[29] G. Grigoryan, Coiled coil crick parameterization, http:// www.grigoryanlab.org/cccp/index.gen.html

[30] G. Grigoryan and W. F. DeGrado, J. Mol. Biol. 405, 1079 (2011).

[31] E. F. Pettersen, T. D. Goddard, C. C. Huang, G. S. Couch, D. M. Greenblatt, E. C. Meng, and T. E. Ferrin, J. Comput. Chem. 25, 1605 (2004).

[32] J. Y. Su, R. S. Hodges, and C. M. Kay, Biochemistry 33,

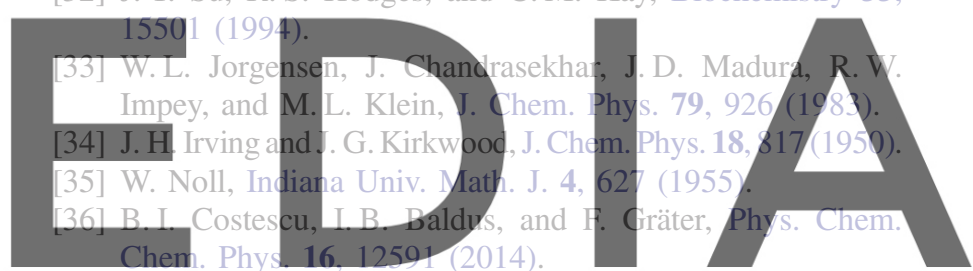

[37] J. M. Vanegas, A. Torres Sánchez, and M. Arroyo, Comput

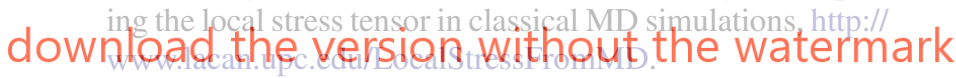

[38] A. I. Murdoch, J. Elast. 71, 105 (2003).

[39] H. Wagner, J. Stat. Phys. 55, 1293 (1989).

[40] A. I. Murdoch, Physical Foundations of Continuum Mechanics, 1st ed. (Cambridge University Press, Cambridge, England, 2012).

[41] B. Seguin and E. Fried, Arch. Ration. Mech. Anal. 206, 1039 (2012).

[42] D. Marsh, Chem. Phys. Lipids 144, 146 (2006).

[43] W. Helfrich, J. Phys. Condens. Matter 6, A79 (1994).

[44] R. G. Littlejohn and M. Reinsch, Rev. Mod. Phys. 69, 213 (1997).

[45] J. Porta, L. Ros, F. Thomas, and C. Torras, IEEE. Trans. Robot. 21, 176 (2005).

[46] L. Mistura, Int. J. Thermophys. 8, 397 (1987).

[47] M. Baus and R. Lovett, Phys. Rev. Lett. 65, 1781 (1990).

[48] G. C. Rossi and M. Testa, J. Chem. Phys. 132, 074902 (2010).

[49] T. Doyle and J. Ericksen, in Advances in Applied Mechanics IV, edited by H. L. Dryden and Th. von Kármán (Elsevier, New York, 1956), Vol. 4, pp. 53115.

[50] J. Marsden and T. J. R. Hughes, Mathematical Foundations of Elasticity (Dover Publications, Inc., New York, 1983).

[51] N. C. Admal, Ph.D. thesis, University of Minnesota, 2014.

[52] A. MacKerell and D. Bashford, J. Phys. Chem. B 102, 3586 (1998).

[53] Z. Zhang, X. Wang, and J. D. Lee, J. Appl. Phys. 115, 114314 (2014). 